\title{
Three-dimensional Electron Ptychography of Catalyst Nanoparticles using Combined HAADF STEM and Atom Counting
}

\author{
Emanuela Liberti ${ }^{1,2^{*}}$, Gerardo T. Martinez ${ }^{1}$, Colum M. O’Leary ${ }^{1}$, Peter D. Nellist ${ }^{1}$ and Angus I. \\ Kirkland $^{1,2}$ \\ 1. University of Oxford, Department of Materials, Oxford, United Kingdom. \\ 2. electron Physical Sciences Imaging Centre (ePSIC), Diamond Light Source, United Kingdom. \\ * Corresponding author: emanuela.liberti@materials.ox.ac.uk
}

High angle annular dark-field (HAADF) imaging in the scanning transmission electron microscope (STEM) is a powerful tool for obtaining structural information of metallic catalyst particles at the atomic scale. Due to recent developments in quantification methods it is now possible to count atoms in individual atomic columns of nanoparticles and hence to estimate their three-dimensional structure from a single image projection [1,2]. These quantification methods exploit the monotonic dependence of the image counts with thickness and as such they are designed ideally for heavy elements, which scatter strongly to the HAADF detector.

The lack of sensitivity towards light elements in incoherent, Z-contrast imaging modes makes current quantification methods unsuitable for counting atoms in non-metallic systems, or metal oxides, to obtain, for example, three-dimensional structural information of heterogeneous oxide catalysts. In contrast, phase imaging techniques are more effective in detecting light elements, but quantification is challenging as the inherent coherent scattering process results in images where contrast is in general not directly interpretable.

Recent advances in STEM electron ptychography have made it possible to obtain quantitative phase information simultaneously with Z-contrast imaging [3]. The development of fast pixelated detectors enables the recording of all scattered beams at the detector plane, at any number of probe positions. Consequently, Z-contrast images can be recovered by integrating electron counts over any combination of inner/outer scattering angles, and simultaneously on a conventional annular dark-field detector. At the same time, electron ptychography can be used to restore the specimen exit wavefunction by recording electron diffraction patterns at every pixel.

The phase of the exit wave contains in principle all structural information from the specimen, including the number of atoms per atomic column and their relative distance (focus) to the exit surface (zinformation) [4]. However, this information can be difficult to quantify due to coherency related artifacts. One way of tackling this problem is to obtain a reliable measurement of the atom count from the simultaneous ADF STEM image. This thickness can subsequently be used as a scaling factor to calibrate absolute phase shifts in the restored phase. Furthermore, structural information along the beam direction can be obtained by restoring the exit wavefunction at a specific depth in the specimen, which can be performed during post-processing. This depth sectioning effect, alongside the atom count, allows for a full three-dimensional reconstruction of the object.

We will present this quantitative approach for three-dimensional structure retrieval of a Pt nanoparticle. Figure 1(a) shows the phase of the specimen wavefunction, restored from a ptychographic reconstruction using the single side-band method [5]. The atom count resulting from the simultaneous 
ADF image (b) is displayed in figure 1(c), alongside a first estimate of the 3D model (d). The retrieval of the three-dimensional structure from the full 4D STEM data, which is achieved by combining the coherent and incoherent information in Figure 1, will be discussed in detail. Preliminary results from ceria nanoparticles, showing the prospect of quantifying stoichiometry alongside atomic structure will also be presented.

\section{References:}

[1] L. Jones et al., Nanolett. 14 (2014), 6336.

[2] S. Van Aert et al., Phys. Rev. B 87 (2013), 064107.

[3] H. Yang et al., Nat. Comm. 7 (2016), 12532.

[4] D. Van Dyck et al., Nature 486 (2012), 243.

[5] H. Yang et al., Ultramic. 151 (2015), 232.

[6] A. De Backer et al., Ultramic. 171 (2016), 104.
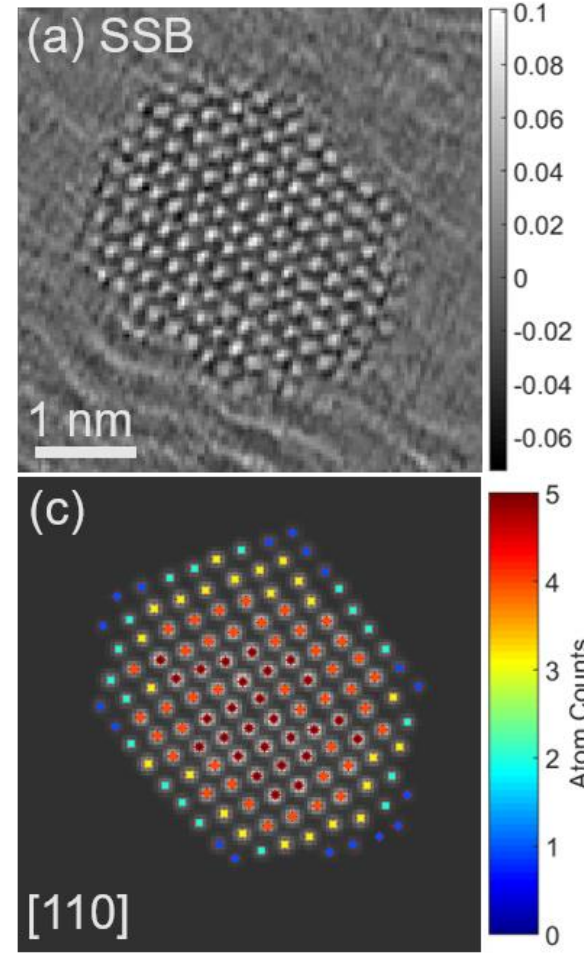

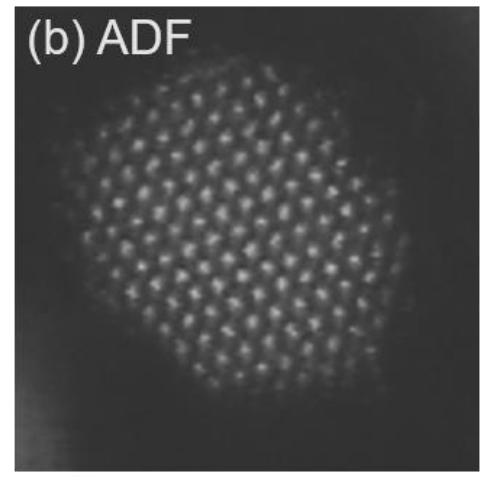

(d)

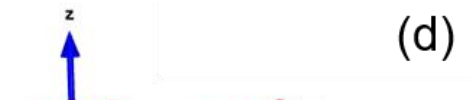

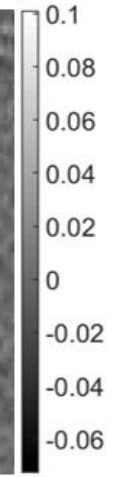

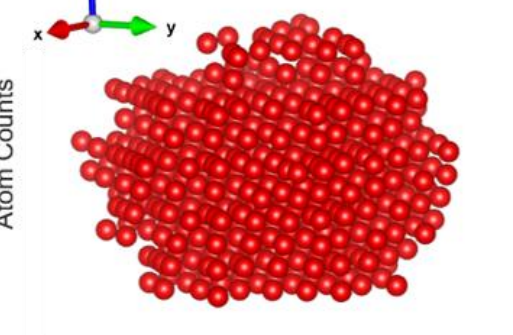

Figure 1. Ptychographic phase (a, colour bar: radians) of a Pt nanoparticle catalyst on a carbon support in a [110] projection, reconstructed using the single side-band approach [5]. The 4D STEM data was acquired simultaneously with the ADF STEM image in (b), which was recorded on a conventional annular detector, with an inner angle of $56 \mathrm{mrad}$ and an outer angle of approximately $200 \mathrm{mrad}$. A Medipix 3 direct electron detector was used to acquire 256 x 256 diffraction patterns, at a speed of 2 $\mathrm{kHz}$. The experiment used a JEOL ARM300CF double-corrected electron microscope, operated at $200 \mathrm{kV}$, with a convergence semi angle of $22 \mathrm{mrad}$. The atom count (c) and the estimated (unrelaxed) three-dimensional model (d) were obtained from the procedure in [6]. 